\title{
Spatial Dimensions of Population Viability
}

Mats Gyllenberg (mats.gyllenberg@utu.fi)

Ilkka Hanski (ilkka.hanski@helsinki.fi)

Johan A.J. Metz (metz@rulsfb.leidenuniv.nl)

\section{Approved by}

Ulf Dieckmann (dieckman@iiasa.ac.at)

Program Leader, Adaptive Dynamics Network

November 2004 


\section{Contents}

1 Introduction 1

2 Deterministic versus Stochastic Metapopulation Models 2

Metapopulations with few patches ................... 2

Metapopulations with many patches $\ldots \ldots \ldots \ldots \ldots$

3 Threshold Phenomena and Basic Reproduction Ratios 3

Basic reproduction ratios and persistence . . . . . . . . . . . . 3

Persistence and viability ......................... 4

4 Modeling Structured Metapopulations 5

Defining the environmental interaction variable . . . . . . . . . . . 6

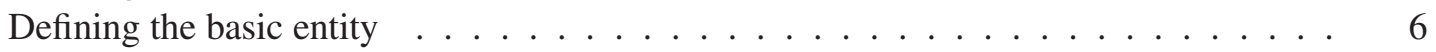

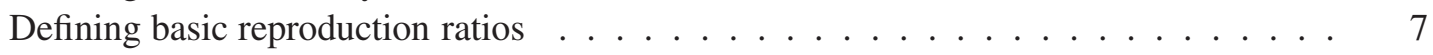

5 Metapopulation Structured by Local Population Density $\quad 8$

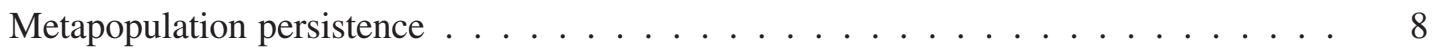

Metapopulation viability . . . . . . . . . . . . . . . . . . . . 10

Toward more realistic models . . . . . . . . . . . . . . . . . . . . . . . . . 11

6 Persistence of Finite Metapopulations: Stochastic Models 11

Predictions from a spatially explicit stochastic model . . . . . . . . . . . . . . 12

New introductions . . . . . . . . . . . . . . . . . . . . . . . . 14

Between stochastic and deterministic models . . . . . . . . . . . . . . . . . . . 14

7 Concluding Comments $\quad 16$ 


\title{
About the Authors
}

\author{
Mats Gyllenberg \\ Department of Mathematics \\ University of Turku \\ FIN-20014 Turku, Finland
}

\author{
Ilkka Hanski \\ Department of Ecology and Systematics \\ University of Helsinki \\ P.O. Box 7, FIN-00014 Helsinki, Finland
}

\author{
Johan A.J. Metz \\ Section Theoretical Biology \\ University of Leiden \\ Kaiserstraat 63, NL-2311 GP Leiden, The Netherlands \\ and \\ Adaptive Dynamics Network \\ International Institute for Applied Systems Analysis \\ A-2361 Laxenburg, Austria
}

\section{Acknowledgments}

The work of Mats Gyllenberg has been supported by the Academy of Finland, and the work of Mats Gyllenberg and Johann A.J. Metz by the European Research Training Network ModLife (Modern Life-history Theory and its Application to the Management of Natural Resources) Network, funded through the Human Potential Programme of the European Commission (Contract HPRN-CT-2000-00051). 


\title{
Spatial Dimensions of Population Viability
}

\author{
Mats Gyllenberg \\ Ilkka Hanski \\ Johan A.J. Metz
}

\section{Introduction}

In most parts of the world, habitat loss is the number one threat to endangered species. For instance, in Finland the primary cause of threat is some form of habitat loss or alteration in $73 \%$ of the red-listed species (Rassi et al. 2001). Typically, a reduced total area of habitat is accompanied by habitat fragmentation, such that the remaining habitat occurs in smaller fragments with reduced connectivity. Many landscapes for many species have become highly fragmented (the habitat fragments are small or relatively small and physically completely isolated), while other landscapes have always been highly fragmented naturally. Species that live in such landscapes necessarily have fragmented populations, which more or less closely approach the metapopulation structure originally envisioned by Levins (1969). Levins' metapopulation is a system of local populations that inhabit individual habitat patches connected, to some extent, by migration. The classic metapopulation concept assumes that local populations may go extinct, and so leave the respective habitat patch temporarily unoccupied, while the metapopulation as a whole may persist in a balance between extinctions and colonizations (Levins 1969; Hanski and Gilpin 1997; Hanski 1999). In a broader sense, any assemblage of local populations connected by migration can be called a metapopulation, regardless of the occurrence of local extinctions (Hanski and Gilpin 1997). What is important is the spatially localized interactions of individuals, which may significantly change the dynamics of the metapopulation as a whole in comparison with a single panmictic population (Hanski 1999).

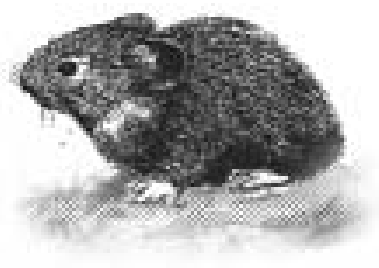

Collared pika Ochotona collaris

The metapopulation concept has received much attention from conservation biologists during the past 15 years (Soulé 1987; Western and Pearl 1989; Falk and Holsinger 1991; McCullough 1996; Young and Clarke 2000), ever since it replaced the dynamic theory of island biogeography as the main population ecological paradigm in conservation biology (Hanski and Simberloff 1997). The number of well-studied examples of species with a distinctive metapopulation structure and frequent local extinctions is increasing rapidly; these include butterflies, mammals (like the American pika), plants, and plant-herbivore-parasitoid communities (reviews in Hanski 1999, 2001). For European butterflies, many of which have suffered greatly from habitat loss and fragmentation (Pullin 1995), tens of studies have demonstrated the critical role of metapopulation processes in setting the condition for their regional persistence, or extinction (Thomas and Hanski 1997; Hanski 1999). One example is the Glanville fritillary butterfly in the Åland Islands in southwest

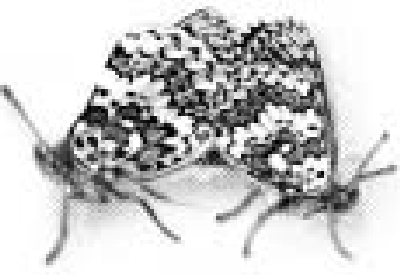

Glanville fritillary butterfly Melitaea cinxia

Finland, which lives in a landscape that is highly fragmented, and probably has been so throughout the period the species has inhabited this area (Hanski 1999). Figure 1 illustrates the pattern 


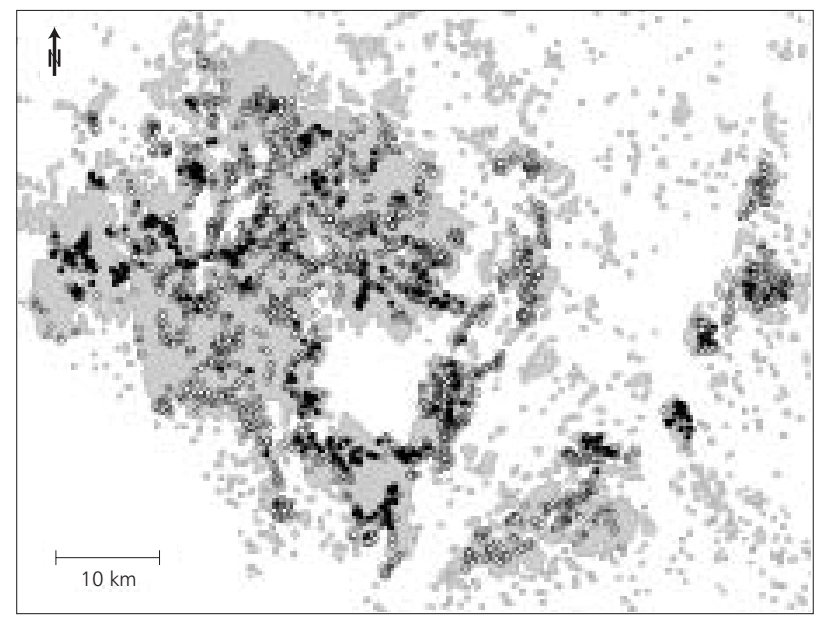

Figure 1 Metapopulation of the Glanville fritillary in the Åland Islands (Finland). The map shows occupied (filled circles) and empty (open circles) suitable habitat patches in autumn 2000.

of habitat patch occupancy in one year, and shows that only some $20 \%$ of the suitable habitat is occupied at any one time. Nonetheless, there is much population turnover, extinctions, and colonizations in this metapopulation, and which particular patches are occupied and which are empty changes continuously in time (Hanski 1999). Research on the Glanville fritillary demonstrates conclusively one of the key messages from the metapopulation theory, namely that the currently empty habitat is as important for long-term persistence as the currently occupied habitat.

\section{Deterministic versus Stochastic Metapopulation Models}

As in other branches of ecology, mathematical modeling has proved an indispensable tool for understanding the dynamics of metapopulations. The choice of the model depends very much on the real-world situation to be modeled. The number, sizes, and locations of the patches and the sizes of the local populations all influence the choice of model.

\section{Metapopulations with few patches}

If both the number of patches and the number of individuals in each patch are small, then the metapopulation must be modeled using stochastic processes (Gyllenberg and Silvestrov 1994, 1999, 2000; Etienne and Heesterbeek 2001; Gyllenberg, in press). In this case the metapopulation becomes extinct on an ecological time scale with the probability of 1 . Therefore, if evolution is to be studied at least one of these numbers must be large. Furthermore, in the limiting case, in which one of these numbers is infinite, the long-term viability and persistence of metapopulations can be dealt with in simple qualitative terms. If all the patches have a large carrying capacity, then a deterministic model describes the local dynamics. If the number of patches is small and there are no local extinctions, then the dynamics at the metapopulation level is described by either a system of finitely many ordinary differential equations (in the continuous-time case) or by a set of finitely many coupled maps (in the case of discrete time). In the continuous-time case the analysis of persistence and viability thus reduces to well-known results from the theory of ordinary differential equations (see, e.g., Hofbauer and Sigmund 1988). The discrete time case still presents some technical difficulties (Gyllenberg et al. 1993) and even some surprising phenomena; for instance, it is possible that the replacement of a good-quality patch by a poor-quality patch may salvage the metapopulation from extinction (Gyllenberg et al. 1996). 


\section{Metapopulations with many patches}

Another possibility is to assume an infinite number of equally coupled patches. This also allows catastrophes to be incorporated in a relatively simple manner. The price to be paid is that it is no longer possible to model explicitly the spatial configuration of the patches. The deterministic metapopulation models treated in this chapter are therefore based on the assumptions that the local populations are internally homogeneously mixed and that the patches are equally coupled. These assumptions may seem unrealistic, but for many purposes, including the calculations in this chapter, a rule of thumb is that these simplifying assumptions can be used with impunity if, in a spatial configuration, each patch is reached easily from more than 20 neighbors. In Section 5 we delve a little deeper into the connection between stochastic reality and our deterministic idealizations of it, and in Section 6 we discuss a stochastic metapopulation model in which the simplifying assumptions mentioned above are not made.

A question of utmost importance is, of course, under what conditions will a metapopulation persist. For instance, what is the minimum amount of habitat that will guarantee metapopulation persistence? How does habitat deterioration affect metapopulation persistence? What is the minimum viable metapopulation size? In stochastic models of metapopulations with a finite number of patches with finite local populations, these questions are replaced by questions about the distribution of the extinction time and, in particular, the expected time to extinction.

\section{Threshold Phenomena and Basic Reproduction Ratios}

It is well-known that persistence of metapopulations is linked to threshold phenomena. We illustrate this with the following slightly modified Levins model, which was first used by Lande (1987) to investigate the effect of the amount of habitat upon metapopulation persistence,

$$
\frac{d p}{d t}=c p(h-p)-\mu p,
$$

where $p$ is the fraction of occupied patches, $c$ is the colonization parameter, $\mu$ is the extinction rate per local population, and $h$ is the fraction of suitable patches. This model is based on the assumption that the colonization rate is proportional to the fraction of occupied patches (from where the potential colonizers come) and the fraction $h-p$ of empty but suitable patches (the patches that can potentially be colonized). A simple calculation shows that the trivial solution that corresponds to metapopulation extinction, that is $p=0$, is the only steady state and is stable if $c h<\mu$. If $c h>\mu$, the extinction equilibrium is unstable and there exists a unique nontrivial steady state. Thus, the dimensionless parameter $R_{0}$, defined by

$$
R_{0}=\frac{c}{\mu} h,
$$

sets a threshold on metapopulation persistence: the metapopulation persists if and only if $R_{0}>1$.

\section{Basic reproduction ratios and persistence}

The quantity $R_{0}$ has a clear-cut and important biological interpretation. It is the expected number of new local populations produced by one local population placed in an otherwise virgin environment, that is in an environment with all other patches empty. The parameter $R_{0}$ is a direct analog of the $R_{0}$ used in epidemic models (Diekmann et al. 1990; Diekmann and Heesterbeek 1999); indeed, Equation (1) is nothing but the celebrated "susceptible-infected-susceptible" (SIS) model, in which the empty patches are the susceptible individuals and the occupied patches are the infected individuals.

When $R_{0}>1$, the nontrivial steady state is immediately obtained in the usual way by putting $d p / d t=0$ in Equation (1) and solving for $p$. However, to set the stage for the coming sections 


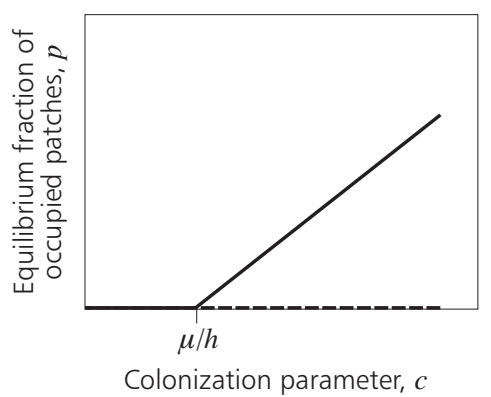

Figure 2 Bifurcation diagram of Levins' model [Equations (1) and (3)], in which the stable and unstable equilibria of $I=p$ are plotted (continous and dashed lines, respectively) against the colonization parameter $c$, while the other parameters $\mu$ and $h$ are kept constant.

we proceed in a slightly more cumbersome, but at the same time more instructive, way. To this end we rewrite the Levins model, Equation (1), as

$$
\frac{d p}{d t}=[c(h-I)-\mu] p,
$$

where we have simply replaced one $p$ in Equation (1) with the symbol $I$. The point of this seemingly meaningless trick is that, assuming $I$ is given, Equation (3) takes the form of a linear differential equation. For the nontrivial steady state $I=p$ is a constant different from 0 . However, according to Equation (3), this is the case when $c(h-I)-\mu=0$, or, equivalently, when the quantity defined by

$$
R_{I}=\frac{c}{\mu}(h-I)
$$

is equal to 1 . Then the equilibrium fraction of occupied patches obtained is $p=h-(\mu / c)$.

The introduction of an auxiliary variable (usually called the environmental interaction variable, denoted by $I$ ) that cuts the feedback loop and makes the model linear, assuming that the variable is a known function of time, is the leitmotif of modeling structured (meta)populations (Metz and de Roos 1992; Gyllenberg et al. 1997; Diekmann et al. 2001). An extra advantage is that the steady state condition is formulated in terms of the quantity $R_{I}$, which has a biological interpretation similar to that of $R_{0}$. It is the expected number of new local populations produced by one local population during its entire life, given that the fraction of occupied patches is $I$. The steady state criterion $R_{I}=1$ thus formalizes the intuitively obvious requirement that a local population on average exactly replaces itself. Note the consistency in the notation: $R_{I}$ goes to $R_{0}$ as $I$ goes to zero.

The results presented above can be summarized and illustrated conveniently by a bifurcation diagram (Figure 2). In Figure $2 c$ is (quite arbitrarily) chosen as the bifurcation parameter. If the expected lifetime $1 / \mu$ of a local population or the fraction $h$ of habitable patches were chosen as the bifurcation parameter, qualitatively similar diagrams would be obtained.

\section{Persistence and viability}

We have introduced the general notion of metapopulation persistence. This, by definition, means that the metapopulation extinction equilibrium is unstable. We have shown that a metapopulation governed by the Levins model is persistent if and only if $R_{0}>1$. Also, whenever the Levins metapopulation is not persistent, the metapopulation inevitably becomes extinct. Therefore, in the case of the Levins model, persistence coincides with another important notion, namely that of viability, which we define in general by the existence of a nontrivial attractor. By "nontrivial" we simply mean "other than the extinction equilibrium"; the "attractor" can be a steady state, a periodic orbit, or even a chaotic attractor, but in this chapter we restrict ourselves to steady states. 


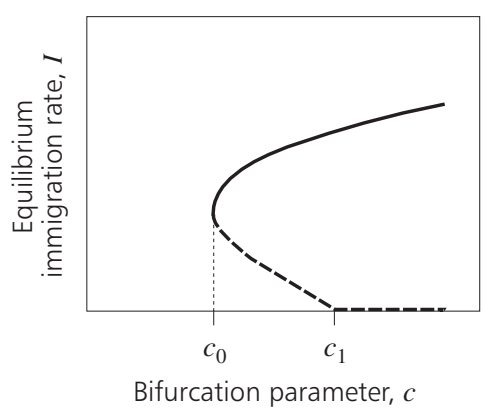

Figure 3 Bifurcation diagram of a hypothetical metapopulation model (solid curve, stable equilibria; dashed curve, unstable equilibria). The metapopulation is persistent for all $c$ values larger than $c_{1}$, and viable for all $c$ values larger than $c_{0}$.

We emphasize that the coincidence of persistence and viability is a peculiarity of the Levins model and need not be true for more complicated metapopulation models. To see this, suppose that a model has a bifurcation diagram as that depicted in Figure 3; an interval of parameter (e.g., c) values occurs in which the metapopulation is viable, but not persistent. In Section 5 we give an explicit example of a metapopulation model in which this situation occurs.

The Levins model, Equation (1), is simplified in the extreme. In particular, the dynamics is modeled directly at the level of the metapopulation, ignoring local dynamics altogether. This is manifested in the interpretation of the basic ingredients or parameters: $\mu$ is the extinction rate per local population and $c$ is the colonization rate per local population and empty patch. Thus, the persistence criterion $R_{0}>1$, with $R_{0}$ given by Equation (2), yields a necessary and sufficient condition for persistence in terms of these parameters (and the parameter $h$ ) at the metapopulation level. There is no obvious relation between $c, \mu$, and the behavior of individuals. However, one of the main goals of evolutionary conservation biology is to understand how population persistence and viability are influenced by individual traits that may be adaptive. To investigate this question we have to turn to more complicated models that explicitly prescribe local dynamics in terms of parameters that describe individual behavior. Such models are called structured metapopulation models and have been treated by, among others, Gyllenberg and Hanski (1992, 1997), Hanski and Gyllenberg (1993), Gyllenberg et al. (1997, 2002), Gyllenberg and Metz (2001), Metz and Gyllenberg (2001), and Parvinen (2001a, 2001b). In Section 4 the persistence and viability of metapopulations is investigated within the context of structured models. The key technique is to define the basic reproduction ratios $R_{0}$ and $R_{I}$ for these models.

\section{Modeling Structured Metapopulations}

Simple, unstructured metapopulation models face severe limitations. The aim to make predictions that relate to real data raises the need to include an explicit description of migration in terms of the numbers of individuals moving, rather than a description restricted to the colonization rate of empty habitat, as in the Levins model and other patch-occupancy models. It is now widely recognized that both emigration and immigration often have important consequences for the dynamics of especially small local populations (Stacey et al. 1997; Thomas and Hanski 1997; Hanski 1998, 1999), and hence also for the dynamics of metapopulations that consist of such small populations. The rescue effect (Brown and Kodric-Brown 1977) - the reduced risk of extinction in a local population because of immigration - is the best-known example of such effects (Hanski 1985), which can be accounted for in a mechanistic manner within the framework of structured models (Gyllenberg and Hanski 1992).

When modeling and analyzing the temporal dynamics of structured populations, the starting point is to describe mechanisms at the individual level, then lift the model to the population level, and finally study phenomena at the population level. As pointed out already by Metz and 
Diekmann (1986) (see also Diekmann et al. 1988, 1989), and later by Gyllenberg and Hanski (1992) (see also Gyllenberg et al. 1997), the theory of structured populations can be applied to metapopulations in a rather straightforward manner if an analogy is made between local populations and individuals and between metapopulation and population. We have seen an instance of this already in our brief discussion of the classic Levins model. However, in some cases a more general analog of an individual is needed, examples of which are given later. The entity of a metapopulation that corresponds to an individual in ordinary populations is called the basic entity.

As a practical aside, note that below we concentrate on the limiting case of infinite, which in practice means large, local populations. These may become extinct as a consequence of a local catastrophe. The deterministic nature of the model means that a patch which becomes empty as a result of a local disaster is immediately recolonized. Therefore, according to the model there are no empty patches (except in the case of metapopulation extinction). Yet in reality empty patches do exist. When fitting the model to data this dichotomy can be overcome of by introducing a detection threshold, by which a patch of local population size less than this threshold is empty.

\section{Defining the environmental interaction variable}

A basic entity develops (i.e., its state changes with time) as a consequence of, for instance, patchquality dynamics, that is the local population growth through births, deaths, and migration. It gives rise to new local entities (e.g., local populations produce dispersers, which colonize empty patches), and some vanish (e.g., when a local population becomes extinct or a disperser dies). To model mechanisms at the local level, two ingredients are needed, one to describe the production of new local entities and one to describe the development and survival of local entities. These ingredients depend on the environmental interaction variable $I$, which must be chosen such that for a given $I$ the model becomes linear. The value of $I$, in turn, depends on the metapopulation state and therefore the full model at the level of the metapopulation becomes nonlinear. We refer to articles by Gyllenberg et al. (1997), Diekmann et al. (2001), Gyllenberg and Metz (2001), and Gyllenberg and Jagers (in press) for accounts of how the modeling task can be carried out in general.

One of the salient features of structured metapopulation models is that they make it possible to model and hence analyze how migration affects local dynamics. Emigration is as easy to model as death is; from the point of view of a local population, it does not make any difference whether an individual is lost through death or emigration. Immigration is more complicated, unless it is assumed that the immigration rate $I$ is known, in which case the modeling task is easy, since $I$ is just an additional contribution to the growth rate of the local population. The immigration rate $I$ depends, of course, on the overall density of dispersers, which in turn depends on the emigration rate and mortality during dispersal. The nonlinear feedback thus takes place through migration, and the immigration rate $I$ qualifies as an environmental interaction variable. Assuming $I$ to be known, a linear problem is obtained, but the true value of $I$ is found by closing the feedback loop.

\section{Defining the basic entity}

The goal of this chapter is to understand the determinants of metapopulation persistence and viability. We had seen already, in connection with the Levins model, that this issue can be investigated using the basic reproduction ratios $R_{0}$ and $R_{I}$. We therefore generalize these quantities to structured metapopulation models. The Levins model, however, is so simple that it is not immediately clear how this should be done. There is a general abstract framework for defining the basic reproduction ratios (Gyllenberg et al. 1997; Diekmann et al. 1998; Gyllenberg and Metz 2001; Metz and Gyllenberg 2001), but here we are content with a more intuitive approach that takes advantage of biological interpretation. Let us first examine how the basic reproduction ratio is interpreted for "ordinary" structured populations, that is, for populations without the metastructure. 
For such models the basic reproduction ratio is the expected number of offspring born to a typical individual during its entire life. Here, part of the problem is to define what the word "typical" means.

For a given individual with a known state at birth, the expected lifetime production of offspring can be calculated from the basic model ingredients, and finding $R_{0}$ and $R_{I}$ then amounts to averaging (in the right way!). To translate the ideas of ordinary populations into metapopulations, we must first define the basic entity. The local population may, at first sight, seem the obvious choice. However, in terms of an evolutionary approach to metapopulation viability, it is important to understand the invasion and fixation of rare mutants that have life-history traits that are different from those of a wild type formerly established in the metapopulation. One therefore has to investigate the competition between different types that inhabit the same local population, and therefore the local population itself does not contain sufficient information to qualify as a basic entity.

We restrict our attention to models with two types of local entities: dispersers and resident clans. A resident clan consists of an individual that arrives at a patch (the "ancestor") and all its descendants (children, grandchildren, great grandchildren, etc.), as long as they stay in the patch. When a resident emigrates we say that the clan gives birth to a disperser. It is convenient to think of the arrival of a disperser to a patch as the simultaneous death of the disperser and the birth of a new resident clan. [Note that "resident", as used in this chapter, means simply an individual who lives in a patch, as opposed to a migrant, and should not be confused with the notion of a resident phenotype as opposed to that of a mutant phenotype in the context of adaptive dynamics theory (see Chapters 11, 14, 16, and 17 in Ferrière et al. 2004).]

Note that in the metapopulation context one cannot base the persistence criterion on an individual-based reproduction ratio. An individual may be very prolific locally, but if the dispersal rate is not large enough almost all of its descendants may be lost at a local disaster. It is therefore necessary to take the resident clan as defined above, and not the individual, as the basic unit of the metapopulation.

\section{Defining basic reproduction ratios}

To calculate the basic reproduction ratio of a structured metapopulation we first have to find an expression for the expected (cumulative) number and local-state distribution of "offspring" produced by a "newborn" basic entity. At this stage we assume that such an expression is well-defined, given the model. Later we discuss how this expression can be obtained from more detailed models of individual behavior.

We make three important assumptions about individual behavior:

- All dispersers behave in the same way, that is, dispersers are unstructured;

- Dispersers choose their new patch at random;

- The behavior of residents may depend on the state $X$ of their local population.

More complicated models could be treated in the same spirit and formally, using the same abstract method (Gyllenberg et al. 1997; Diekmann et al. 2001). The following ingredients can now specify the model:

- $E_{I}(X)$, the expected number of dispersers produced by a clan that was initiated by a disperser immigrating into a local population of state $X$, given that the immigration rate is $I$;

- $\phi$, the probability that a disperser survives migration and starts a new clan.

With these model specifications, the expected number of new clans produced by a clan initiated by a disperser that arrives at a local population of state $X$ is $\phi E_{I}(X)$. We assume dispersers choose their new patch at random and therefore the state-at-birth of new clans equals the steady population size distribution $p_{I}$ that corresponds to the immigration rate $I$. A "typical" clan is therefore one sampled from the steady population size distribution $p_{I}$ and we obtain Equation (5a) 
for the reproduction ratio of basic entities,

$$
R_{I}=\phi \int E_{I}(X) p_{I}(X) d X,
$$

where the integral is taken over all possible local population states $X$.

The steady population size distribution $p_{I}$ depends on the local dynamics, which are not yet specified. It can be shown (Gyllenberg et al., unpublished) that at equilibrium the basic reproduction ratio becomes

$$
R_{I}=\phi \frac{E_{I}}{I \ell_{I}},
$$

where $E_{I}$ is the expected number of offspring produced by a local population during its entire life and $\ell_{I}$ is the expected lifetime of a local population, given the immigration rate $I$. This formula has a very intuitive interpretation. Note that the denominator on the right-hand side is the expected number of arrivals at a patch. The whole right-hand side is therefore the expected number of arriving (anywhere in the metapopulation) offspring of a local population divided by the mean number of arrivals at a patch. At equilibrium, this quantity should equal 1 , hence $R_{I}$. It can also be shown that the equilibrium value of $R_{I}$ given by Equation (5b) tends to $R_{0}$ as the immigration rate $I$ tends to zero (Gyllenberg et al. 2002).

\section{Metapopulation Structured by Local Population Density}

In this section we illustrate our theory by a simple example which has the population density, denoted as $N$, as the local state. Our model is specified by the following ingredients:

- $h$, the fraction of habitable patches;

- $r(N)$, the density-dependent per capita growth rate from local births and deaths;

- $m(N)$, the density-dependent per capita emigration rate;

- $\phi$, the probability that a disperser survives migration and establishes a new clan;

- $\mu(N)$, the density-dependent local catastrophe rate.

This enables persistence and viability to be expressed using the expected number $E_{I}$ of dispersers produced by a local population during its entire life, the expected lifetime $\ell_{I}$ of a population, and Equation (5b) to obtain $R_{I}$ at equilibrium, taking the limit as $I$ tends to zero to obtain $R_{0}$. This was carried out by Gyllenberg et al. (2002). However, to calculate $R_{0}$ there is a shortcut, which we shall follow.

\section{Metapopulation persistence}

Consider a newly founded resident clan in an otherwise virgin environment. This means that all other patches are empty and that the local population of the clan is size zero; as a consequence, so long as the metapopulation remains small we can neglect the effects of density dependence. Thus, the local per capita growth rate, per capita emigration rate, and catastrophe rate are constant and equal to $r(0), m(0)$, and $\mu(0)$, respectively. Necessary and sufficient conditions of persistence are derived in Box 1. Results can be summarized as follows:

- If local growth at zero density is slow enough, namely if $r(0)$ is less than the sum $m(0)+$ $\mu(0)$, then the persistence criterion is

$$
R_{0}=h \phi \frac{m(0)}{m(0)-[r(0)-\mu(0)]}>1 .
$$


Box 1 Deriving a criterion for structured metapopulation persistence

Here we use the model assumptions and notations of Section 5 to present a derivation of the persistence criterion based on $R_{0}$. We consider a newly founded resident clan in an otherwise virgin environment. The probability that this clan is still extant $t$ time units later is $\exp [-\mu(0) t]$, and if it is extant its size is $\exp (-[r(0)-m(0)] t)$. Each of the individuals in the clan has a probability $m(0) d t$ of migrating in the infinitesimal time interval $[t, t+d t]$. Summing over all times, we obtain the expected number of migrants produced by a clan, denoted by $E_{0}(0)$,

$$
E_{0}(0)=\int_{0}^{\infty} m(0) \exp ([r(0)-m(0)-\mu(0)] t) d t=\frac{m(0)}{\mu(0)+m(0)-r(0)} .
$$

Note that the symbol $E_{0}(0)$ is consistent with the notations used so far: the subscript 0 indicates zero immigration and the argument 0 means that the clan starts from an empty patch, all in accordance with the notion of a virgin environment.

The equality in Equation (a) is, of course, valid if and only if $r(0)<m(0)+\mu(0)$, otherwise the integral is infinite [unless $m(0)=0$, in which case $E_{0}$ assumes the value 0 ]. We accept the possibility that $E_{0}$ will take on infinite values and adopt the usual arithmetic on $[0, \infty]$, including the convention $0 \times \infty=0$.

From the interpretation of the quantities involved it is clear that $R_{0}=\phi E_{0}(0) h$, but we emphasize that this can be rigorously deduced from Equation (5a) since the steady population size distribution $p_{I}$ that corresponds to the population-free case is the point mass of size $h$ concentrated at the origin.

Persistence, then, is found to be determined by the relative values $r(0)$ and $m(0)+\mu(0)$ :

- If $r(0)<m(0)+\mu(0)$, the persistence criterion given by Equation (6) is obtained.

- If $r(0)>m(0)+\mu(0)$ and $m(0)>0$, then $R_{0}=\infty$ if $h>0$, and $R_{0}=0$ if $h=0$. Thus, in this case $R_{0}>1$ if and only if $h>0$.

This persistence criterion is very similar to the corresponding criterion for the Levins model. There is an important difference, though. Whereas the latter was formulated in terms of the parameters $c$ and $\mu$, which measure attributes at the local population level, Equation (6) contains the per capita growth and emigration rates $r(0)$ and $m(0)$, which are properties of individuals.

- If local growth at zero density is fast enough, namely if $r(0)$ is larger than $m(0)+\mu(0)$, and if the emigration rate at low density $m(0)$ is not null, then the persistence criterion $R_{0}>1$ is equivalent to $h>0$. This entails that fast local growth can compensate for arbitrary loss of habitat and keep the metapopulation alive - any positive amount of suitable habitat is enough to ensure persistence. Notice that in Levins' model, which neglects local dynamics, nothing of this sort is feasible.

Focusing on the specific effect of the emigration rate on metapopulation persistence, two further cases may be distinguished when local growth is slow $[r(0)<m(0)+\mu(0)]$ :

- If local growth is too slow the metapopulation is not persistent regardless of the emigration rate, namely, if $r(0) \leq \mu(0)$;

- For intermediate rates of local growth, i.e., $\mu(0) \leq r(0)<m(0)+\mu(0)$, the metapopulation persistence requires that emigration be less than an upper threshold, denoted by $m_{1}$ [obtained by rearranging terms in Equation (6)]. This threshold $m_{1}$ increases with the difference between the rates of local growth and catastrophe at low density, the probability of survival and establishment for dispersers, and the amount of suitable habitat. 
Box 2 Deriving a criterion for structured metapopulation viability

Here we provide the analysis of metapopulation viability in the context of the structured model introduced in Section 5. To this end, we have to calculate $R_{I}$ at equilibrium by using Equation (5a). First, observe that our specification of individual behavior implies that local population growth is governed by the differential equation

$$
\frac{d N}{d t}=r(N) N-e(N) N+I .
$$

Now, consider a local population at the time of a local disaster and simultaneous recolonization. The probability that it is still extant when it has density $N$ is equal to $\exp \left(-\int_{0}^{N} \frac{\mu(x)}{r(x) x-m(x) x+I} d x\right)$, and given that it is extant with density $N$ the expected number of dispersers produced in the infinitesimal density interval $[N, N+d N]$ is $\frac{m(N) N}{r(N) N-m(N) N+I} d N$. The factor $1 / r(N) N-m(N) N+I$ that occurs in these formulas simply reflects a conversion from quantities per unit of time to quantities per unit of population density. Adding up over all sizes gives

$$
E_{I}=\int \frac{m(N) N}{r(N) N-m(N) N+I} \exp \left(-\int_{0}^{N} \frac{\mu(x)}{r(x) x-m(x) x+I} d x\right) d N .
$$

Similarly, one obtains

$$
\ell_{I}=\int \frac{1}{r(N) N-m(N) N+I} \exp \left(-\int_{0}^{N} \frac{\mu(x)}{r(x) x-m(x) x+I} d x\right) d N .
$$

The expression for $R_{I}$ at equilibrium follows readily from Equation (5a).

\section{Metapopulation viability}

At the metapopulation steady state the condition $R_{I}=1$ holds (see Box 2), and Figure 4 shows plots of the equilibrium fraction of occupied patches determined by this condition as a function of the emigration rate (from Gyllenberg et al. 2002). It may be a surprise that, even for $r(0) \leq$ $\mu(0)$, in which case the metapopulation is not persistent, there might very well exist a range of emigration rates $m(0)$ over which the metapopulation is viable. This is possible even in the case of density-independent migration (i.e., with constant $m$ ), and can be seen in Figures $4 \mathrm{~b}$ and $4 \mathrm{c}$ : in the range $m_{1}<m<m_{2}$ there exists a stable nontrivial equilibrium and hence the metapopulation is viable for migration rates in this range. In Figures $4 \mathrm{a}$ and $4 \mathrm{~b}$ the condition $\mu(0)<r(0)$ is satisfied, and we know from the previous subsection that there is a range $0<m<m_{1}$ above which the metapopulation is persistent. If the catastrophe rate is constant (Figure 4a), then persistence and viability coincide. If, on the other hand, the catastrophe rate is a decreasing function of local population density (Figures $4 \mathrm{~b}$ and $4 \mathrm{c}$ ), there is a range $m_{1}<m<m_{2}$ of emigration rates for which the metapopulation is viable but not persistent.

These results illustrate the possibility of alternative locally stable equilibria in metapopulation dynamics (see also Hanski 1985; Hanski and Gyllenberg 1993; Gyllenberg et al. 1997). The size of the metapopulation may move from the domain of one stable equilibrium to the domain of the alternative equilibrium following a large environmental perturbation, and at the bifurcation point the metapopulation is predicted to show a deterministic "jump" from the nontrivial equilibrium to metapopulation extinction. The lesson here is that it should not be assumed that slight changes in parameter values will necessarily be reflected in only slight changes in metapopulation size, and it is possible that large long-lasting changes in metapopulation size will occur in response to small environmental perturbations. The feedback between migration and local dynamics, on the one hand, and the dynamics of the entire metapopulation, on the other, may generate discontinuous changes in the abundance and distribution of species. 

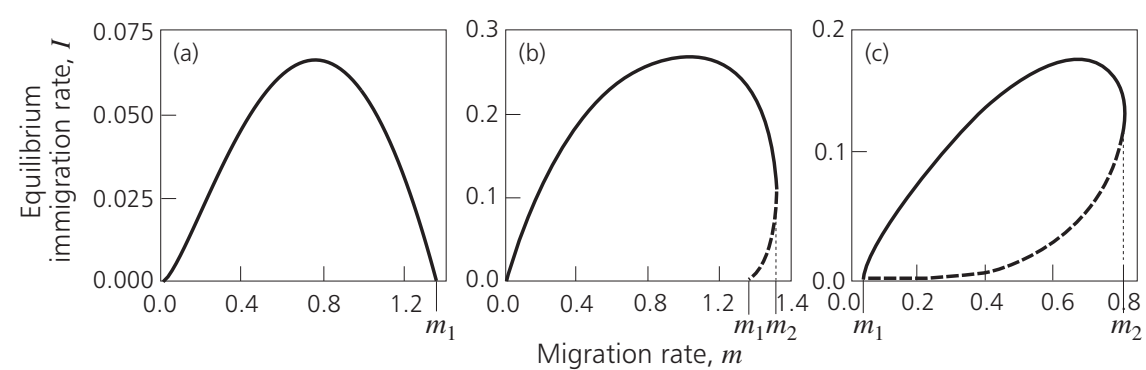

Figure 4 Bifurcation diagrams for the structured metapopulation model described in Section 5. The stable and unstable equilibria values of $I$ are plotted against the per capita migration rate $m$, which is density independent and acts as bifurcation parameter. (a) Constant rate of catastrophe: $\mu=0.4$. (b) The catastrophe rate $\mu(N)$ decreases with increasing local population density $N$, and $\mu(0)=0.4$. (c) The catastrophe rate $\mu(N)$ is as in (b), but with $\mu(0)=1.2$. In all cases the local population growth is logistic, with $r(N)=1-N, \phi=0.55$, and $h=1$.

\section{Toward more realistic models}

The structured metapopulation models analyzed in this section omit a description of the spatial population structure in that they assume the habitat patches are identical (though see Gyllenberg and Hanski 1997) and equally connected. This is not a great restriction for systems that consist of large networks of well-connected habitat patches without a strongly aggregated spatial distribution; yet to relate the modeling results to empirical studies it is often useful to account explicitly for the spatial structure in metapopulations. The spatially structured metapopulation models developed by Hanski $(1994,1999)$ have recently been analyzed mathematically by Hanski and Ovaskainen (2000) and Ovaskainen and Hanski (2001, 2002, unpublished). This line of modeling retains the present or absent description of dynamics in local populations, as in the original Levins model, but allows for finite patch networks with differences in the areas, qualities, and degree of connectivity of the patches. An advantage of these models is that they can be given rigorous parameters for real metapopulations (ter Braak et al. 1998; Moilanen 1999, 2000), and therefore establish a firmer link between theory and empirical studies (Hanski 1999).

However, once again, because individuals are not modeled explicitly, the spatially structured models cannot be extended easily to evolutionary studies without resorting to individual-based simulations. [For an example see Heino and Hanski (2001), who combined individual-based simulations with a spatially structured patch occupancy model to constrain the model-predicted long-term dynamics and used a statistical model of individual movement behavior to model migration of individuals among multiple populations.] One challenge for further research is to develop metapopulation models that include both the spatial structure and the local population size structure.

\section{Persistence of Finite Metapopulations: Stochastic Models}

So far we have considered metapopulations that consist of infinitely many patches, which, moreover, in the structured case contain infinitely many individuals. The reason for doing so is that the results for such models can be stated in simple intuitive terms, that we have good tools for studying them, and that we may expect the results to hold to a good approximation when we replace "infinite" with "sufficiently large". Of course, real populations are not infinite. In this section, dedicated to predict relationships between individual traits and metapopulation viability on the basis of stochastic models, we first treat a particular example of a finite metapopulation model and clearly illustrate the concepts involved in a nontrivial manner. We follow this up with a heuristic overview of how the mathematically idealized infinite cases connect to the more realistic finite cases. 


\section{Predictions from a spatially explicit stochastic model}

To exemplify the main concepts that occur in population models of a more finite kind, we concentrate on a stochastic metapopulation model with a finite number of patches. The spatial arrangements of patches is modeled explicitly. The basic ideas and results are most easily described when time is taken as a discrete variable, and therefore from here onward we switch to discrete-time models. Our model is based on Gyllenberg (in press), but also see Etienne and Heesterbeek (2001). Analogous, but technically more difficult, results for a continuous-time stochastic metapopulation model were derived by Gyllenberg and Silvestrov [1999, 2000; see also Frank and Wissel (1994, $1998,2002)$ for practically useful heuristics]. We choose this model structure, which is rather different from those considered previously, because it allows us to show some overarching ideas and to elucidate some of the interpretational problems that occur when comparing models of different origin.

We consider a collection of $n$ patches that can be either occupied or empty at the discrete time instants $t=0,1,2, \ldots$ Metapopulation extinction corresponds to all patches being empty. The local dynamics are modeled by preassigning an interaction matrix $Q=\left[q_{j i}\right]$ in which:

- $q_{i i}(i=1,2, \ldots, n)$ is the probability that, in the absence of migration, the population inhabiting patch $i$ will become extinct in one time-step;

- $q_{j i}(i=1,2, \ldots, n, j=1,2, \ldots, n$, and $j \neq i)$ is the probability that patch $i$ will not be colonized in one time-step by a migrant originating from patch $j$.

Typically, $q_{j i}$ depends on at least the distance between the patches $i$ and $j$ and the area of patch $j$. This model incorporates the notion of a rescue effect, that is, the decreasing extinction rate with increasing fraction of occupied patches. The overall extinction probability of the local population that inhabits patch $i$ may be considerably less than the "internal" extinction probability $q_{i i}$ if there are many large occupied patches in the vicinity (many small $q_{j i}$ ).

The analysis of this model (see Box 3) requires three conditions:

- No local population is able to colonize another patch in one time-step with probability 1 ;

- Even in the absence of migration (rescue effect), no local population has extinction probability 1 and no local population is protected from extinction;

- Every local population is able to colonize any other patch either directly or through a chain of patches (stepping-stone dispersal).

These conditions together imply that we are, indeed, dealing with a true metapopulation and not, for instance, with a mainland-island model, in which only migration from the mainland to the islands is allowed, or with a collection of several disconnected metapopulations.

Since there is a finite number of patches, sooner or later all extant populations will simultaneously become extinct and the whole metapopulation will be wiped out. Mathematically speaking, the metapopulation will become extinct with probability 1 . In such cases of certain extinction, there is no stationary distribution except the trivial one that corresponds to metapopulation extinction. However, we can define the so-called quasi-stationary distribution (Darroch and Seneta 1965; see also Chapter 2 in Ferrière et al. 2004), which is the stationary distribution on the condition that the metapopulation has not become extinct.

If we consider a metapopulation that has been extant for a considerable time, we may use the quasi-stationary distribution as the starting point from which to consider the time to its future extinction; we refer to the corresponding time to extinction as the quasi-stationary extinction time. This solves the problem that, in general, the time to future extinction is highly dependent on the state in which the metapopulation is at present. In particular, we have to distinguish situations such as a reintroduction from situations in which the metapopulation has been present for a long time. Subject to some natural monotonicity conditions, if a metapopulation has known more favorable 
Box 3 A spatially explicit stochastic model

We consider a collection of $n$ patches that can be either occupied or empty at the discrete time instants $t=0,1,2, \ldots$, and make use of the notations introduced in Section 6 to analyze the metapopulation dynamics. The state of patch $i$ at time $t$ is given by the random indicator variable $\eta_{i}(t)$, which takes on the value 1 if patch $i$ is occupied and 0 if patch $i$ is empty at time $t$. The state of the metapopulation is described by the vector random process in discrete time $\eta(t)=\left(\eta_{1}(t), \eta_{2}(t), \ldots, \eta_{n}(t)\right)$, with $t=0,1,2 \ldots$. The state space of the process $\eta(t)$ is $\Xi=\left\{\xi=\left(x_{1}, \ldots, x_{n}\right): x_{i} \in\{0,1\}\right\}$. It has $2^{n}$ states. The state $\mathrm{O}=(0, \ldots, 0)$ corresponds to metapopulation extinction.

We assume that the local extinction processes and the colonization attempts from different local populations are all independent. As a consequence of this independence the conditional probabilities $q_{i}(\xi)$ for patch $i$ to be empty at time $t+1$, given that at time $t$ the metapopulation was in state $\xi=\left(x_{1}, \ldots, x_{n}\right)$, are given by the product

$$
q_{i}(\xi)=\prod_{j=1}^{n} q_{j i}^{x_{j}}, \quad i=1,2, \ldots, n,
$$

where we use the convention $0^{0}=1$.

Having described the local patch dynamics, we can deduce the law that governs the time evolution of the process $\eta(t)$ and gives the state of the metapopulation. This process is a homogeneous Markov chain with state space $\Xi$ and transition probabilities

$$
\prod_{i=1}^{n} q_{i}(\xi)^{1-y_{i}}\left[1-q_{i}(\xi)\right]^{y_{i}}, \quad \xi, \zeta \in \Xi .
$$

The process $\eta(t)$ is determined completely by the interaction matrix $Q$, which is assumed to satisfy the following conditions (stated verbally in the text of Section 6):

$q_{j i}>0, j \neq i$

- $0<q_{i i}<1, i \in\{1, \ldots, n\}$

- For each pair $(j, i)$ of patches, $j, i \in\{1, \ldots, n\}$, there exists an integer $l$ and a chain of indices $j=i_{0}, i_{1}, \ldots, i_{l}=i$ such that $\prod_{k=1}^{l}\left(1-q_{i_{k-1} i_{k}}\right)>0$.

The process can be described in terms of its quasi-stationary distribution, which is given by the left eigenvector (normalized to a probability distribution) that corresponds to the dominant eigenvalue of the transition matrix $Q$ restricted to the transient class $\Xi \backslash O$. The dominant eigenvalue measures the probability that a metapopulation sampled from the quasi-stationary distribution will not become extinct in one time step.

conditions in the past, the time to extinction under a constant continuation of the current regime will be larger (but usually not much) than the quasi-stationary one. Conversely, if a metapopulation has just been started from a few individuals, the time to extinction will be less than the quasistationary extinction time. (For the mathematically inclined reader, here "below" and "above" should be interpreted as inequalities on the corresponding survival functions.)

Conditional on nonextinction, the state distribution will approach the quasi-stationary distribution. It therefore makes sense to view the dynamics of the metapopulation as a two-state Markov process, the states being metapopulation extinction and the quasi-stationary distribution. If $q$ denotes the probability that a metapopulation sampled from the quasi-stationary distribution will become extinct in one time step, the expected extinction time is equal to $1 / q$. This is an exact expression for the expected time to extinction, provided that the metapopulation is initially at the quasi-stationary distribution. However, the exact calculation of $q$ (which is obtained as the eigenvalue of a $2^{n}-1$ by $2^{n}-1$ matrix) becomes computationally prohibitive as the number of patches $n$ grows. For the continuous-time case, a good approximation can be found in Frank and Wissel (2002). We refer to Etienne and Heesterbeek (2001) for examples of how this result can be applied 
to reach practical conclusions about, for instance, how changing the connectivity of patches, that is changing the $q_{j i}$ values, affects the viability of the metapopulation.

\section{New introductions}

As a second consideration, we look at new introductions. In this case the probability of becoming extinct in the next time step is, in general, larger than $q$, and only decreases to $q$ in the long run. The general rule-of-thumb is that in systems with more or less global coupling, $q$ increases to 1 with increasing system size (see below for a further elaboration), while the time needed for the stepwise extinction probability to converge to $q$ increases much more slowly than $1 / q$, the mean extinction time after reaching quasistationarity. This means that for larger system sizes we may consider the probability distribution of the time to extinction as consisting of a discrete mass at zero followed by an exponential tail. In those cases that have global coupling, it is in general possible to calculate the initial mass from a branching process approximation. For example, if each patch may contain at most one individual, then under the assumptions of the Levins model (see Section 3) the initial mass equals approximately $[\mu /(c h)]^{\Omega}$ if $\mu<c h$, and 1 (so that there is no tail left) if $\mu>c h$, where $\Omega$ is the number of individuals that start the metapopulation. More generally, for the deterministic models considered in Sections 3 to 5, the initial mass can be written as $\theta^{\Omega}$, where $\theta$ equals 1 when $R_{0} \leq 1$, and $\theta<1$ when $R_{0}>1$. However, these results have the caveat that it is assumed implicitly that females may always reproduce. Many real populations of conservation interest contain two sexes. This means that, even though there are many individuals, if they all happen to be of one sex a population may have no future. Whether such considerations really matter greatly depends on the detailed reproductive biology of the species (see Chapters 2 and 3 in Ferrière et al. 2004 for a discussion of this issue in the context of nonspatial models). Further research into this area should be both a mathematical and a biological priority.

\section{Between stochastic and deterministic models}

The main difference between population models with finite total numbers of individuals and those in which these number are thought of as infinite is that in the former the population, in the long run, becomes extinct whatever the value of the parameters. However, the time for this to happen is generally very long when the number of "close-to-independent" entities involved is large. This latter number is referred generally to as the system size. In metapopulation models, as considered here, there are actually two system sizes, the number of patches $n$ and the patch size $\omega$ (the latter is expressed in units roughly equal to the amount of space needed to support a single individual). We have to consider their interplay to determine what sort of limit is obtained and to establish the scaling relations between the extinction time and system sizes, when either or both of them become large.

By the argument of the above subsection, when system sizes become large, the mean extinction time of a population starting from a nonvanishing population differs from the mean quasistationary extinction time only by a relatively small amount. It is these quasistationary mean extinction times that we discuss below. For brevity, we refer to them simply as extinction times.

To establish a feel for the problem, we first consider how the transition to the deterministic model is made for a single local population, without considering immigration from other patches and catastrophes. Let $N$ be the number of individuals in the patch and the rates at which these individuals die, give birth, or emigrate as, respectively, $b(N / \omega), d(N / \omega)$, and $m(N / \omega)$, where the unit of the patch size $\omega$ and functions $b, d$, and $m$ be such that $b(1)-d(1)-m(1)=0$, that is, the equilibrium density of a deterministic population model based on those functions equals 1 . With this scaling we can identify the local system size with $\omega$. We obtain a deterministic limit model for the temporal development of the local population density $N / \omega$ by letting $\omega$ become large. (The 
limit holds good over any finite time interval, but not over the full time axis, except for those cases in which the deterministic model predicts certain extinction, since for all finite $\omega$ the population becomes extinct if we wait long enough.) In addition, according to accepted wisdom, "in viable systems the extinction time of an established local population is roughly exponential in the system size". More precisely, the extinction time, $T_{\mathrm{p}}$, scales exponentially with $\omega$, written as $T_{\mathrm{p}} \approx \mathrm{e}^{\theta \omega}$, to be interpreted as $\frac{1}{\omega} \ln T_{\mathrm{p}} \rightarrow \theta$ for $\omega \rightarrow \infty$, in which $\theta$ is the so-called scaling constant. This result has been shown to hold good in the simplest possible models (MacArthur 1972; see also the figures in Goodman 1987b), is shored up by arguments from statistical physics [see, e.g., Gardiner (1983); also, Schuss (1980), Grasman and HilleRisLambers (1997), Freidlin and Wentzell (1998), and Grasman and Van Herwaarden (1999), who specifically consider metapopulation problems], and is backed up by simulations for some other models. With catastrophes, for large $\omega$ the local population size develops deterministically until the first catastrophe, while the extinction time, $T_{\mathrm{p}}$, is set by the catastrophe rate.

Next, assume that we have $n$ similar patches equally coupled through migration, and that we let the local system size $\omega$ become large. We can then make more than one biologically meaningful assumption about the immigration rate.

One such assumption is that the migration parameters are constants, so that at larger patch sizes the number of immigrants grows directly in proportion to $\omega$. Translated into observable quantities, this assumption implies that at least some patches should contain reasonable numbers of individuals and that the average interarrival time of immigrants is small relative to the mean lifetime of individuals. If the local population growth rates decrease with density, and there are no catastrophes, we have a fully deterministic model in which all patches in the long run contain an equal population density; this is positive if the quantity $R_{0}$, calculated according to the analysis in the previous section, is larger than 1 . If there are catastrophes, the metapopulation may die out even in the limit for large $\omega$, but it may continue forever. The former definitely happens when $R_{0} \leq 1$, or the parameter domain that leads to extinction is always larger, with its size shrinking with increasing $n$, than that given by the deterministic criterion $R_{0} \leq 1$ (unpublished results by ourselves).

Another possibility is to change the migration parameters such that the mean interarrival time of immigrants is of the same order of magnitude as the mean lifetime of a local population, $T_{\mathrm{p}}$. This limit regime applies when the probability of surviving migration and reaching some other patch is of the order of $\omega^{-1}$, or when emigration is relatively rare on a patch basis, or a combination of both these factors. At the same time, we assume that the catastrophes occur at a rate that is slow compared to the speed at which quasistationarity would be reached by immigrationless local populations. Moreover, we consider the metapopulation on the time scale set by $T_{\mathrm{p}}$. (Despite the somewhat artificial look of the mathematical procedure, the required parameter regime may well be rather common in nature because parameters for community assemblage and selection setting are in a commensurable range.) In this case, patches are either empty or in a quasi-stationary state almost all of the time, and we have a finite Levins-type model, provided the local populations cut off from immigration have persistent deterministic limits (compare Verboom et al. 1991; Drechsler and Wissel 1997). If we now let $n$ become large, while keeping the migration rate into the patches bounded, we recover the deterministic Levins model from Section 3, with $\mu=1 / T_{\mathrm{p}}$. The time to metapopulation extinction, $T_{\mathrm{m}}$, scales linearly with $T_{\mathrm{p}}$ and exponentially with $n$. In this case we even have available a full asymptotic formula [by applying Stirling's approximation to Equation (6) in Frank and Wissel 2002], applicable for $R_{0}=c T_{\mathrm{p}}=c / \mu>1$,

$$
T_{\mathrm{m}} \approx T_{\mathrm{p}} \sqrt{2 \pi} n^{-1 / 2} \mathrm{e}^{\left[\left(\mathrm{c} T_{\mathrm{p}}\right)^{-1}-1+\ln \mathrm{c} T_{\mathrm{p}}\right](n-1)} .
$$

For $R_{0}<1$, the average extinction time of a metapopulation starting from any positive fraction of occupied patches increases logarithmically with $n$. 
By taking the Levins-type models as a gauging point, we can now write for persistent metapopulation models

$$
T_{\mathrm{m}}=T_{\mathrm{p}} \psi(\omega, n) n^{-1 / 2} \mathrm{e}^{\gamma n},
$$

with the function $\psi$ thus defined as measuring the rescue effect. Of course, both $\gamma$ and $\psi$ depend on all the other system parameters, as well as on how the migration and catastrophe rates are supposed to scale with $\omega$ and $n$. However, we conjecture that for any relevant scaling, $\omega$ will be nondecreasing, at least at larger system sizes, because the reliability of the migration stream increases as system size increases.

To show the potential force of the rescue effect, we can compare a Levins model without catastrophes (so that $T_{\mathrm{p}} \approx \mathrm{e}^{\theta \omega}$ ) with a model in which migration increases so fast with $\omega$ or $n$ that, effectively, all patches can be considered together as one single population. Combining the relationships found above then gives $\psi(\omega, n) \approx \mathrm{e}^{\kappa \omega n} /\left(\mathrm{e}^{\gamma n} \mathrm{e}^{\gamma n}\right) \approx \mathrm{e}^{\kappa \omega n}$ for $\omega$ and $n$ both large, that is, the rescue effect overwhelms all other contributions to the scaling of $T_{\mathrm{p}}$. However, the example in which we keep $n$ constant and let $\omega$ become large at a constant per capita migration rate makes clear that the limit behavior of the rescue effect can be more complicated than in the example given in this paragraph.

In the above discussion, we implicitly referred to the case where, in the deterministic limit model, the metapopulation is persistent. The case in which the metapopulations are viable, but not persistent, is less clear. However, we also expect an exponential scaling with $n$ (by a rough appeal to the arguments found in Schuss 1980; Gardiner 1983; Freidlin and Wentzell 1998; Grasman and Van Herwaarden 1999). This with the proviso that we expect the scaling constant $\gamma$ to be roughly proportional to the distance of the equilibrium population state of the deterministic model from the closest point in the state space of that model from where, in the deterministic model, the state would move inexorably toward extinction (where nearness is measured in terms of the "ease of a state transition").

\section{Concluding Comments}

In this chapter the stress is on modeling migration and local dynamics at the individual level rather than at that of local populations. One reason for carefully analyzing the limit relationships between different types of metapopulation models is that this allows us to interpret each of these models from an individual-level perspective. Only in this way can we give a concrete meaning to the model parameters. Achieving such a concreteness is the first step on the arduous path that leads from model results to conservation interventions.

In the chapter we strive to stay within the realm of what is manageable with present-day mathematical methods, while going one step further in the interpretation process than our predecessors. We are well aware that the models we discuss are considerably less concrete than individual- or GIS-based simulation models that purportedly mimic the behavior of specific species. However, even those of our colleagues who believe that we can render a fair fraction of such detailed models right agree that we cannot model all the species we ultimately have to deal with. So we are in dire need of good rules of thumb. It is here that we may hope that the simpler models of this chapter and their future extensions will prove useful.

The second advantage of basing our models on individual-level considerations is the possibility that evolutionary questions, such as the evolution of migration rate, could be addressed (for reviews see Clobert et al. 2001; Ferrière et al. 2000; Gyllenberg and Metz 2001; Metz and Gyllenberg 2001; Parvinen 2001b; see also Chapter 14 in Ferrière et al. 2004). This is simply not possible with patch-occupancy models [see Hanski (1999) for a review], except, perhaps, in the restricted sense of selection that occurs at the level of local populations (group selection). Further merging 
of ecological and evolutionary dynamics in the context of structured metapopulation models is an exciting prospect for modeling, and one of considerable importance if we are to gain at least a little grip on the potential long-term consequences of human-induced environmental change.

\section{References}

Brown JH \& Kodric-Brown A (1977). Turnover rates in insular biogeography: Effect of immigration on extinction. Ecology 58:445-449

Clobert J, Danchin E, Dhondt AA \& Nichols JD (2001). Dispersal. Oxford, UK: Oxford University Press

Darroch J \& Seneta E (1965). On quasi-stationary distributions in absorbing discrete-time finite Markov chains. Journal of Applied Probability 2:88-100

Diekmann O \& Heesterbeek JAP (1999). Mathematical Epidemiology of Infectious Diseases: Model Building, Analysis and Interpretation. New York, NY, USA: John Wiley and Sons

Diekmann O, Metz JAJ \& Sabelis MW (1988). Mathematical models of predator-prey-plant interactions in a patchy environment. Experimental and Applied Acarology 5:319-342

Diekmann O, Metz JAJ \& Sabelis MW (1989). Reflections and calculations on a prey-predatorpatch problem. Acta Applicandae Mathematicae 14:23-25

Diekmann O, Heesterbeek JAP \& Metz JAJ (1990). On the definition of the basic reproduction ratio $R_{0}$ in models for infectious diseases in heterogeneous populations. Journal of Mathematical Biology 28:365-382

Diekmann O, Gyllenberg M, Metz JAJ \& Thieme HR (1998). On the formulation and analysis of general deterministic structured population models. I. Linear theory. Journal of Mathematical Biology 36:349-388

Diekmann O, Gyllenberg M, Huang H, Kirkilionis M, Metz JAJ, \& Thieme HR (2001). On the formulation and analysis of general deterministic structured population models. II. Nonlinear theory. Journal of Mathematical Biology 43:157-189

Drechsler M \& Wissel C (1997). Separability of local and regional dynamics in metapopulations. Theoretical Population Biology 51:9-21

Etienne RS \& Heesterbeek JAP (2001). Rules of thumb for conservation of metapopulations based on a stochastic winking-patch model. The American Naturalist 158:389-407

Falk DA \& Holsinger KE (1991). Genetics and Conservation of Rare Plants. Oxford, UK: Oxford University Press

Ferrière R, Belthoff JR, Olivieri I \& Krackow S (2000). Evolving dispersal: Where to go next? Trends in Ecology and Evolution 15:5-7

Ferrière R, Dieckmann U \& Couvet D (2004). Evolutionary Conservation Biology. Cambridge, UK: Cambridge University Press.

Frank K \& Wissel C (1994). Ein Modell über den Einfluss räumlicher Aspekte auf das Überleben von Metapopulationen. Verhandlungen der Gesellschaft für Ökologie 23:303-310

Frank K \& Wissel C (1998). Spatial aspects of metapopulation survival: From model results to rules of thumb for landscape management. Landscape Ecology 13:363-379

Frank K \& Wissel C (2002). A formula for the mean lifetime of metapopulations in heterogeneous landscapes. The American Naturalist 159:530-552

Freidlin MI \& Wentzell AD (1998). Random Perturbations of Dynamical Systems, Second Edition. New York, NY, USA: Springer-Verlag

Gardiner CW (1983). Handbook of Stochastic Methods for Physics, Chemistry and the Natural Sciences. Berlin, Germany: Springer-Verlag

Goodman D (1987b). The demography of chance extinction. In Viable Populations for Conservation, ed. Soulé ME, pp. 11-34. Cambridge, UK: Cambridge University Press 
Grasman J \& HilleRisLambers R (1997). On local extinction in a metapopulation. Ecological Modelling 103:71-80

Grasman J \& Van Herwaarden OA (1999). Asymptotic Methods for the Fokker-Planck Equation and the Exit Problem in Applications. Berlin, Germany: Springer-Verlag

Gyllenberg M. Metapopulations. In Branching Processes in Biology: The Ramifications of Life and Death, eds. Jagers P \& Haccou P. Cambridge, UK: Cambridge University Press. In press

Gyllenberg M \& Hanski I (1992). Single-species metapopulation dynamics: A structured model. Theoretical Population Biology 42:35-62

Gyllenberg M \& Hanski I (1997). Habitat deterioration, habitat destruction and metapopulation persistence in a heterogeneous landscape. Theoretical Population Biology 52:198-215

Gyllenberg M \& Jagers P. Branching processes and structured population dynamics. In Branching Processes in Biology: The Ramifications of Life and Death, eds. Jagers P \& Haccou P. Cambridge, UK: Cambridge University Press. In press

Gyllenberg M \& Metz JAJ (2001). On fitness in structured metapopulations. Journal of Mathematical Biology 43:545-560

Gyllenberg M \& Silvestrov DS (1994). Quasi-stationary distributions of a stochastic metapopulation model. Journal of Mathematical Biology 33:35-70

Gyllenberg M \& Silvestrov DS (1999). Quasi-stationary phenomena for semi-Markov processes. In Semi-Markov Models and Applications, eds. Janssen J \& Limnios N, pp. 33-60. Dordrecht, Netherlands: Kluwer Academic Publishers

Gyllenberg M \& Silvestrov DS (2000). Nonlinearly perturbed regenerative processes and pseudostationary phenomena for stochastic systems. Stochastic Processes and Their Applications 86:1-27

Gyllenberg M, Söderbacka G \& Ericsson S (1993). Does migration stabilize local population dynamics? Analysis of a discrete metapopulation model. Mathematical Biosciences 118:2549

Gyllenberg M, Osipov AV \& Söderbacka G (1996). Bifurcation analysis of a metapopulation model with sources and sinks. Journal of Nonlinear Science 6:329-366

Gyllenberg M, Hanski I \& Hastings A (1997). Structured metapopulation models. In Metapopulation Dynamics: Ecology, Genetics and Evolution, eds. Hanski I \& Gilpin M, pp. 93-122. London, UK: Academic Press

Gyllenberg M, Parvinen K \& Dieckmann U (2002). Evolutionary suicide and evolution of dispersal in structured metapopulations. Journal of Mathematical Biology 45:79-105

Gyllenberg M, Metz JAJ \& Parvinen K. A basic reproduction ratio for structured metapopulations. Unpublished

Hanski I (1985). Single-species spatial dynamics may contribute to long-term rarity and commonness. Ecology 66:335-343

Hanski I (1994). A practical model of metapopulation dynamics. Journal of Animal Ecology 63:151-162

Hanski I (1998). Metapopulation dynamics. Nature 396:41-49

Hanski I (1999). Metapopulation Ecology. Oxford, UK: Oxford University Press

Hanski I (2001). Spatially realistic theory of metapopulation ecology. Naturwissenschaften 88:372-381

Hanski I \& Gilpin M (1997). Metapopulation Dynamics: Ecology, Genetics and Evolution. London, UK: Academic Press

Hanski I \& Gyllenberg M (1993). Two general metapopulation models and the core-satellite species hypothesis. The American Naturalist 142:17-41 
Hanski I \& Ovaskainen O (2000). The metapopulation capacity of a fragmented landscape. Nature 404:756-758

Hanski I \& Simberloff D (1997). The metapopulation approach, its history, conceptual domain and application to conservation. In Metapopulation Biology, eds. Hanski I \& Gilpin ME, pp. 5-26. San Diego, CA, USA: Academic Press

Heino M \& Hanski I (2001). Evolution of migration rate in a spatially realistic metapopulation model. The American Naturalist 157:495-511

Hofbauer J \& Sigmund K (1988). The Theory of Evolution and Dynamical Systems. Cambridge, UK: Cambridge University Press

Lande R (1987). Extinction thresholds in demographic models of territorial populations. The American Naturalist 130:624-635

Levins R (1969). Some demographic and genetic consequences of environmental heterogeneity for biological control. Bulletin of the Entomological Society of America 15:237-240

MacArthur RH (1972). Geographical Ecology: Patterns in the Distribution of Species. New York, NY, USA: Harper and Row

McCullough DR, ed. (1996). Metapopulations and Wildlife Conservation. Washington, DC, USA: Island Press

Metz JAJ \& de Roos AM (1992). The role of physiologically structured population models within a general individual-based modeling perspective. In Individual-based Models and Approaches in Ecology, eds. DeAngelis DL \& Gross LJ, pp. 88-111. Routledge, UK: Chapman \& Hall

Metz JAJ \& Diekmann O (1986). The Dynamics of Physiologically Structured Populations, Lecture Notes in Biomathematics, Vol. 68. Berlin, Germany: Springer-Verlag

Metz JAJ \& Gyllenberg M (2001). How should we define fitness in structured metapopulation models? Including an application for the calculation of evolutionarily stable dispersal strategies. Proceedings of the Royal Society of London B 268:499-508

Moilanen A (1999). Patch occupancy models of metapopulation dynamics: Efficient parameter estimation using implicit statistical inference. Ecology 80:1031-1043

Moilanen A (2000). The equilibrium assumption in estimating the parameters of metapopulation models. Journal of Animal Ecology 69:143-153

Ovaskainen O \& Hanski I (2001). Spatially structured metapopulation models: Global and local assessment of metapopulation capacity. Theoretical Population Biology 60:281-302

Ovaskainen O \& Hanski I (2002). Transient dynamics in metapopulation response to perturbation. Theoretical Population Biology 61:285-295

Ovaskainen O \& Hanski I. How much does an individual habitat fragment contribute to metapopulation dynamics and persistence? Unpublished

Parvinen K (2001a). Evolutionary Branching of Dispersal Strategies in Structured Metapopulations. TUCS Technical Report 399. Turku, Finland: Turku Centre for Computer Science

Parvinen K (2001b). Adaptive Metapopulation Dynamics. PhD Thesis, Turku, Finland: University of Turku

Pullin AS, ed. (1995). Ecology and Conservation of Butterflies. London, UK: Chapman \& Hall

Rassi P, Alanen A, Kanerva T \& Mannerkoski I (2001). Suomen lajien uhanalaisuus 2000 [The red-listed species of Finland in 2000]. Helsinki, Finland: Ympäristöministeriö \& Suomen ympäristökeskus

Schuss Z (1980). Theory and Applications of Stochastic Differential Equations. New York, NY, USA: John Wiley and Sons

Soulé ME (1987). Viable Populations for Conservation. Cambridge, UK: Cambridge University Press 
Stacey PB, Johnson VA \& Taper ML (1997). Migration within metapopulations: The impact upon local population dynamics. In Metapopulation Biology, eds. Hanski I \& Gilpin ME, pp. 267-292. San Diego, CA, USA: Academic Press

ter Braak JF, Hanski IA \& Verboom J (1998). The incidence function approach to modeling of metapopulation dynamics. In Modeling Spatiotemporal Dynamics in Ecology, eds. Bascompte J \& Solé RV, pp. 167-188. New York, NY, USA: Springer-Verlag

Thomas CD \& Hanski I (1997). Butterfly metapopulations. In Metapopulation Biology, eds. Hanski I \& Gilpin ME, pp. 359-386. San Diego, CA, USA: Academic Press

Verboom J, Lankester K \& Metz JAJ (1991). Linking local and regional dynamics in stochastic metapopulation models. Biological Journal of the Linnean Society 42:39-55

Western D \& Pearl M (1989). Conservation Biology in the 21st Century. Oxford, UK: Oxford University Press

Young AG \& Clarke GM, eds. (2000). Genetics, Demography and Viability of Fragmented Populations. Cambridge, UK: Cambridge University Press 